\title{
1 Assessing extracellular vesicles from bovine mammary gland 2 epithelial cells cultured in FBS-free medium
}

\author{
3 G. Silvestrelli ${ }^{1}$, S.E. Ulbrich ${ }^{1}$, M.D. Saenz-de-Juano ${ }^{1 *}$ \\ 1) ETH Zurich, Animal Physiology, Institute of Agricultural Sciences, 8092 Zurich, Switzerland. \\ 5 * Correspondence to: M.D Saenz de Juano, ETH Zurich, Animal Physiology, Institute of Agricultural Sciences, 8092 \\ Zurich, Switzerland. E-mail: mara.saenz@usys.ethz.ch ORCID: 0000-0002-2860-2252
}

\section{Abstract}

8 Aim: Mammary gland extracellular vesicles (EVs) are found in both human and livestock milk. Our 9 knowledge of the role of EVs in the mammary gland development, breast cancer and mastitis derives mainly from in vitro cell culture models. However, a commonly shared limitation is the use of foetal bovine serum (FBS) as a supplement, which naturally contains EVs. For this reason, the purpose of the study is to establish a novel tool to investigate mammary gland EVs in vitro and in an FBS-free system.

Methods: Primary bovine mammary epithelial cells (pbMECs) and a mammary gland alveolar epithelial cell line (MAC-T) were cultured in a chemically defined EV-free medium. To find a reliable EVs isolation protocol from a starting cell conditioned medium $(10 \mathrm{~mL})$, we compared eight different methodologies by combining ultracentrifugation (UC), chemical precipitation (CP), size exclusion chromatography (SEC), and ultrafiltration (UF).

Results: The medium formula sustained both pbMECs and MAC-T cell growth and did not alter MAC-T cell identity. Transmission electron microscopy revealed that we obtained EV-like particles in five out of eight protocols. The cleanest samples with the highest particles amount and detectable amounts of RNA were obtained by using UF-SEC-UC and UC-SEC-UC. and allows the isolation of EVs that are free from any contamination by UF-SEC-UC and UC-SEC-UC. In conclusion, we propose a new culture system and EVs isolation protocols for further research on mammary epithelial EVs. 
Mammary gland extracellular vesicles (EVs) participate in many physiological processes of the mammary gland such as development [1,2] and regulation of epithelial cells polarity [3]. They are likewise involved in pathological conditions, including mastitis $[4,5]$ and breast cancer in primis, where EVs are implicated in its onset [6], metastasis [7] and drug resistance [8,9]. Mammary gland EVs are also found in the milk of human [10] and many livestock species such as cattle [11, 12], buffalo [13] and goat [14].

Our knowledge of the molecular mechanisms underlying EVs biogenesis, release, uptake, and effect on recipient cells, derives mainly from in vitro cell culture models. Many studies on human cell lines have investigated the role of EVs in mammary gland communication in the context of cancer $[9,15,16]$ or the maintenance of epithelial cells polarity [3]. In mice, EVs play an important role in mammary gland development [3] and involution [2], and EVs promote the recognition and clearance of apoptotic bodies from macrophages.

While most of the published studies focus on humans and mice, only a few studies investigated EVs directly in bovine mammary gland culture models, from primary cells [17] or cell lines [18]. Bovine milk EVs are heterogenous and comprehend many EVs subtypes including exosomes (40-100 nm) and microvesicles (100-1000 nm). In addition, milk EVs are biologically active, they are transferred to the newborn and are taken up from intestinal cells [11]. To date, their role is still not known in cell-cell communication within the alveolus of the mammary gland. Besides, the origin of milk EVs is not fully clear, despite recent publications suggesting that they are produced by the milk-secreting epithelial cells, the lactocytes $[19,20]$. Of note, autocrine and paracrine communication within the bovine mammary gland alveolus is important in all its developmental steps [21], and, in this frame, EVs might also participate in this intense communication.

Cell culture systems allow more options and flexibility to investigate EVs mediated communication as compared to in vivo systems. However, they usually share a limitation, namely the use of foetal bovine serum (FBS) as a supplement. FBS naturally contains EVs that can interfere with the experimental setting and outcome [22], therefore many studies use commercial or home-made EV-depleted FBS, also in bovine MECs-EVs research [23]. However the removal of EVs from FBS is never complete [22] and alters the FBS effect on cells, affecting cell growth [24], differentiation [25], and response to pathogens [26, 27]. A period of 24-48 hours of starvation from FBS affects the culture conditions as well, leading to cell cycle 
arrest [28] and reducing the background production of cytokines [29], which can cause misinterpretation of cells phenotype and experimental outcome. For these reasons, it is recommended to culture cells directly in FBS-free systems and chemically defined media. Besides the type of medium, it is critical to collect enough EVs for downstream analyses. For this reason, the volume of the starting material usually ranges between 20 and $250 \mathrm{~mL}$ [30-32]. Another factor affecting the yield and purity of the EVs sample is the isolation procedure [33].

Few cell lines from the bovine mammary gland exist, with BME-UV1 [34] and MAC-T [35] as the most commonly known to date. The use of pure epithelial cell lines prevents contamination from fibroblasts often occurring in primary cultures, which can be also avoided by pre-plating [36], short trypsinization and isolating epithelial cells from milk [37]. MAC-T cells are considered a proper model for mammary gland development and lactation [38], as they maintain the capacity to express milk proteins [39] and are responsive to hormonal stimulation [34]. However, cell lines tend to lose the phenotypes of the original tissue [40] and they are set to grow in specific culture conditions, therefore the change of culture conditions can also alter their characteristics.

To gain novel insights on the EVs local communication of the bovine mammary gland in vitro models compiling the guidelines for EVs research are in need. For this reason, in the current study we i) developed a chemically defined FBS-free culture that supports the growth of both primary MEC and MAC-T cell line and ii) compared eight different EVs isolation protocols from only $10 \mathrm{~mL}$ of conditioned medium regarding quantity and size distribution of EVs for further downstream analyses.

\section{METHODS}

\section{Primary cells isolation and culture}

Mammary glands from lactating cows were collected at a local slaughterhouse post mortem and transported on crushed ice to the lab. Only mammary glands that did not display fibrosis, abnormal cell growth, and signs of mastitis (e.g. redness or hardness) were used to set the cultures. Tissue pieces of $\sim 10 \mathrm{~g}$ were pooled from 2 to 4 cows each and washed in ethanol 70\%, then in cold PBS (Gibco, Thermo Fisher, USA) with antibiotics. Tissues were further minced in $\sim 2 \mathrm{~mm} 3$ pieces and washed 6 times in cold PBS with antibiotics. They were digested in Collagenase IV (Sigma-Aldrich, USA) 0,5 mg/mL, Dispase II (Sigma) 0,5 mg/mL, Insulin $5 \mu \mathrm{g} / \mathrm{mL}$ (Sigma), antibiotics and antimycotics in HBSS (Gibco) buffer for 2 hours at $37^{\circ} \mathrm{C}$ while 
gently shaking. The suspension was filtered through a metal mesh to remove larger tissue pieces and then centrifuged at $500 \mathrm{xg}$ for 5 minutes. The pellet was washed twice in PBS and cells were seeded on Nunclon Delta surface dishes (Thermo Fisher) and kept at $37.5{ }^{\circ} \mathrm{C} 7 \% \mathrm{CO}$. For the pre-plating, freshly isolated cells were plated and let for 1 hour in the incubator, then the medium and cells that were not attached yet were transferred into a new plate. The medium was changed every $2-3$ days until $80 \%$ confluence, cells were then subpassaged every 3-4 days. Cells were kept in DMEM/F12 (Gibco), containing Gentamicin 50 $\mu \mathrm{g} / \mathrm{mL}$ (Sigma), Amphotericin B 2,5 $\mu \mathrm{g} / \mathrm{mL}$ (Sigma) and supplemented with a) FBS 10\%, or b) B27 1:50 (Gibco), $5 \mu \mathrm{g} / \mathrm{mL}$ Insulin (Sigma), $5 \mu \mathrm{g} / \mathrm{mL}$ Hydrocortisone (Sigma), Estradiol (E2) $100 \mathrm{nM}$ (Sigma), Progesterone (P4) 300 pM (Sigma) and 5 ng/mL Epidermal Growth Factor (EGF) (Sigma). The latter medium is referred to as FBS-free medium. When plates were coated, rat tail collagen I (Invitrogen, USA) at the final concentration of 6 or $10 \mu \mathrm{g} / \mathrm{cm} 2$, or laminin (Sigma) 1 or $2 \mu \mathrm{g} / \mathrm{cm} 2$ was used. For the growth curve, 6 × 105 cells were seeded on 6-well multiwell plates and counted every two days from day 4 to day 14. To evaluate the growth, $6 \times 105$ cells were seeded on 6-well multiwell plates and counted every two days from day 4 to day 14 using Trypan blue (Sigma) and a Neubauer chamber.

\section{MAC-T cell line}

MAC-T cells were kindly provided by Olga Wellnitz from the Vetsuisse Faculty of the University of Bern (Switzerland). Cells were cultured in FBS 10\% or FBS-free medium and were passaged every 3-4 days. After 2 weeks of adaptation in the FBS-free medium, they were lysed in TRIzol (Thermo Fisher) for checking cell type markers (keratin 18, keratin 14, vimentin) expression. For growth rate evaluation, $1 \mathrm{x}$ 105 cells were plated and counted at $80 \%$ confluence for two consecutive passages (referred to P1 and P2). The growth rate was calculated as $\ln \left(\right.$ cells $\mathrm{t}_{0} /$ cells $\left._{1}\right) / \mathrm{t}_{1}-\mathrm{t}_{0}$.

\section{RNA isolation and retrotranscription}

Once cells reached 80-90\% confluency, they were washed in PBS and lysed in TRIzol (Thermo Fisher), followed by phenol-chloroform RNA isolation. DNA was removed by DNAse I treatment (Sigma). For each sample, $500 \mathrm{ng}$ of total RNA were reverse transcribed using the GoScript Reverse transcription system kit (Promega, USA), following manufacturer instructions and cDNA samples were then stored at $20^{\circ} \mathrm{C}$ until further use. 


\section{Gene expression analysis}

113 Table 1 shows the primer pairs, actin, GAPDH, and histone H3 were used as reference genes. For the RT-

114 qPCR the Kappa Mix (Sigma) was used and the primers concentration was $10 \mu \mathrm{M}$. The amplification was

115 performed using $500 \mathrm{ng}$ of cDNA and the following amplification program: $95^{\circ} \mathrm{C} 3^{\prime}$ (x 1$), 95^{\circ} \mathrm{C} 4^{\prime}, 60^{\circ} \mathrm{C}$

$11620^{\prime}, 95^{\circ} \mathrm{C} 10^{\prime}(\mathrm{x} 40)$. Relative gene expression analysis was performed using the $2-\Delta \Delta \mathrm{Ct}$ method [40].

117

\begin{tabular}{lll} 
Gene & Forward $\mathbf{5} \rightarrow \mathbf{3}$ & Reverse $\mathbf{5} \rightarrow \mathbf{3}$ \\
\hline Actin & GTCTTCCCGTCCATCGTG & TCTTGCTCTGAGCCTCATCC \\
\hline Histone H3 & ACTGGCTACAAAAGCCGCTC & ACTTGCCTCCTGCAAAGCAC \\
\hline GAPDH & GGTCACCAGGGCTGCTTTTA & CCAGCATCACCCCACTTGAT \\
\hline Keratin 18 (KRT18) & ATTTCAGTCTTGGCGACGCT & GCCTCAGTGCCTCAGAACTT \\
\hline Vimentin (VIM) & CGCTCAAAGGGACTAACGAG & ACGAGCCATCTCTTCCTTCA \\
\hline Keratin 14 (KRT14) & CAGCCCCTACTTCAAGACCA & AGGTTCAGCTCCGTCTCGTA \\
\hline Prolactin hormone & CTTGAAAGGAAGCCAAACAGGC & TGGAGAGAATCAACACCGCC \\
receptor (PRLR) & & \\
\hline
\end{tabular}

\begin{tabular}{lll}
\hline Progesterone receptor & GGGACTCTCAGTTCACTTTCAA & TTGTCTGAGTACACGGTGGG
\end{tabular}

(PR)

Alpha casein GGAAGCTGAAAGCATTCGT GGGCACATCTTCCTTTTGAA

CSN1S1

Kappa casein (CSN3) TGCAATGATGAAGAGTTTTTTCCTAG GATTGGGATATATTTGGCTATTTTGT

118 Table 1. List of the designed forward and reverse primer pairs, KRT14 and PRLR are from Finot et al. 2018 [41]

\section{Differential centrifugation (DC)}

The conditioned medium (CM) from cells cultured in FBS-free conditions was collected at 80-90\%

121 confluency. The $\mathrm{CM}$ was differentially centrifuged at $300 \mathrm{xg}, 1000 \mathrm{xg}, 12000 \mathrm{xg} 10 \mathrm{~min}$ at $4^{\circ} \mathrm{C}$ to remove

122 dead cells, cells debris and apoptotic bodies, respectively. Immediately, the $\mathrm{CM}$ was frozen at $-80^{\circ} \mathrm{C}$ until

123 the EV isolation. We combined different methods to isolate EV from $10 \mathrm{~mL}$ of conditioned medium, testing

124 in total 8 routes which are schematized in figure 1 . We started from $0,5 \mathrm{~mL}$ for SEC-UC. 


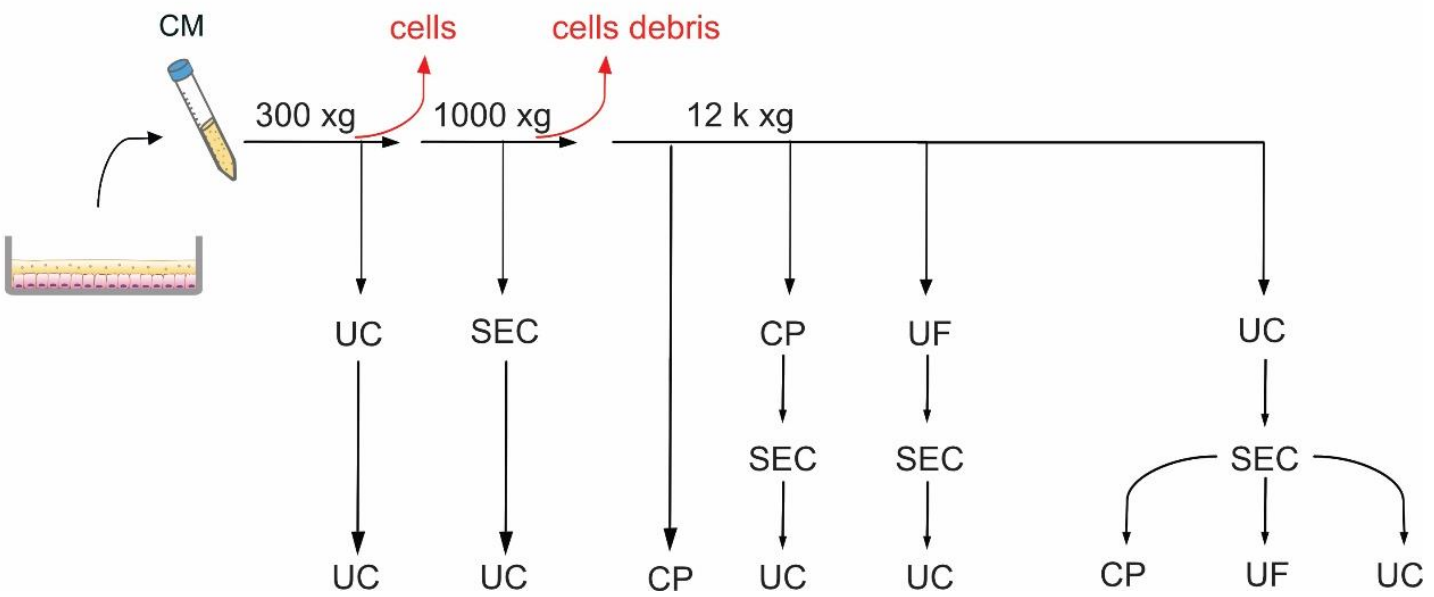

Figure 1. Schematic representation of the 8 protocols (routes) tested. UC: ultracentrifugation, UF: ultrafiltration with Amicon tubes, CP: chemical precipitation with miRCURY (Qiagen), SEC: size exclusion chromatography with qEV columns (IZON).

\section{Ultracentrifugation (UC)}

The $\mathrm{CM}$ was thawed on ice and transferred into $13.5 \mathrm{~mL}$ Ultra-Clear ultracentrifuge tubes (Beckman Coulter, USA). Samples were spun at $120 \mathrm{k} x \mathrm{x}$ for 2 hours or $200 \mathrm{k} \mathrm{xg}$ for $70 \mathrm{~min}$ at $4^{\circ} \mathrm{C}$ in a Beckman Coulter Ultracentrifuge Optima XE-90, using a Type 50.2 Ti rotor. The pellets were resuspended in 0.22 $\mu \mathrm{m}$ filtered PBS for further ultracentrifugation, or size exclusion chromatography, and stored at $-80^{\circ} \mathrm{C}$.

\section{Ultrafiltration (UF)}

To perform UF, $10 \mathrm{~mL}$ of CM or qEV fractions 6-10 diluted in PBS were loaded on $15 \mathrm{~mL}$ Amicon Tubes with a cut-off of $100 \mathrm{kDa}$ (Merck, Germany) and spun for 1 hour RT at $5000 \mathrm{xg}$ on a FA-45-6-30 fixed angle rotor. The concentrate was transferred into a new tube and either frozen or loaded onto a qEV column (IZON Science Ltd, New Zealand).

\section{Precipitation with miRCURY (Qiagen)}

141 To chemically precipitate the EVs, we used the mirCURY kit (Qiagen, Germany) for cell culture medium,

142 following the manufacturer instructions. Briefly, the $\mathrm{CM}$ after differential centrifugation or from size exclusion chromatography was spun at $3000 \mathrm{xg}$ at $4{ }^{\circ} \mathrm{C}$ for 5 minutes to remove debris and cryo-precipitated particles. The supernatant was transferred into a new tube where 0.4 of sample volume of buffer B was added. After vortexing, the tubes were left on ice for 1 hour and then spun at $20^{\circ} \mathrm{C}$ at $3200 \mathrm{xg}$ for 30 
minutes. The precipitate was resuspended in $100 \mu \mathrm{L}$ of resuspension buffer and either snap-frozen or loaded onto qEV columns.

\section{Size exclusion chromatography (SEC)}

We performed size exclusion chromatography with qEV $70 \mathrm{~nm}$ classic columns (IZON). The columns were first equilibrated at room temperature (RT) with $10 \mathrm{~mL}$ of $0.22 \mu \mathrm{m}$ filtered PBS (Gibco), then 500 $\mu \mathrm{L}$ of $\mathrm{CM}$ or EV resuspension from UC or UF was loaded on the column and eluted in filtered PBS. The flow-through was collected in $500 \mu \mathrm{L}$ fractions and in each fraction the protein concentration was measured by the A280 at the Nanodrop. Fractions 6-10 were pulled together for further concentration.

\section{Tunable resistive pulse sensing (TRPS) measurements}

All measurements were conducted using a qNano Gold (IZON) and NP150 polyurethane nanopores (IZON), that detects particles with a diameter ranging from 70 to $420 \mathrm{~nm}$. Filtered PBS (Gibco) was used as an electrolyte buffer and CPC100 (IZON) as calibration particles. Analyses were performed with Izon Control Suite v.3.3.

\section{Transmission Electron Microscopy (TEM)}

The EVs visualization was performed at the Scientific Center for Optical and Electron Microscopy (ScopEM) service of ETH Zurich. Briefly, $3 \mu \mathrm{L}$ of the vortexed dispersion were placed on glow discharged carbon-coated grids (Quantifoil, D) for 1 minute. Negative contrast staining was done in $2 \%$ sodium phosphotungstate pH 7.2 for 1 second, followed by a second step for 15 seconds. Excess moisture was drained with filter paper and the imaging of the air-dried grids was done in a TEM Morgagni 268 (Thermo Fisher) operated at $100 \mathrm{kV}$. For each experimental group, two replicates were analysed.

\section{Proteins isolation and Western blot analysis}

The pellet of freshly isolated EVs from $45 \mathrm{~mL}$ of medium was immediately lysed in RIPA buffer plus protease inhibitors and then stored at $-80^{\circ} \mathrm{C}$. To each sample, $4 \mathrm{x}$ Laemmli buffer (Biorad, USA) was added and heated for $10 \mathrm{~min}$ at $95^{\circ} \mathrm{C}$. Between 1-3 $\mu \mathrm{g}$ of proteins (for bMEC EV, milk EVs, and cell lysates) were run on $12 \%$ polyacrylamide gel and then total protein was evaluated at the Chemidoc running the stain-free program. Proteins were transferred using a TransTurbo transfer pack (Biorad) with a TurboBlot (Biorad). Membranes were blocked 1 hour in skim milk 5\% TBS-Tween buffer (TBST, Bio-Rad, 0.05\% 

secondary antibodies (Santa Cruz Biotechnology, USA) and StrepTactin-AP Conjugate (Biorad) at the concentration of 1:10000, eventually incubated with Clarity Western ECL Substrate (Biorad) for chemiluminescent signal development.

RNA isolation

Total RNA including microRNA (miRNA) were isolated with the miRNeasy MicroKit (Qiagen, Germany). The concentration was measured with Quantus ${ }^{\mathrm{TM}}$ Fluorometer and the QuantiFluor ${ }^{\circledR}$ RNA System kit (Promega). The length from RNA fragments was evaluated using the Agilent Pico Kit and the Agilent 2100 BioAnalyzer (Agilent Technologies).

\section{RNA retrotranscription and $\mathrm{RT}-\mathrm{qPCR}$}

For each sample, $10 \mathrm{ng}$ of RNA were retrotranscribed and pre-amplified using the TaqMan ${ }^{\mathrm{TM}}$ Advanced miRNA assays (Life Technologies), following manufacturer instructions. Then, we performed an RTqPCR using the kit TaqMan ${ }^{\mathrm{TM}}$ Advanced miRNA assays (478575_mir assay), targeting the miR-let7a-5p. The amplification program was: $95^{\circ} \mathrm{C} 30^{\prime \prime}(\mathrm{x} 1), 95^{\circ} \mathrm{C} 5^{\prime}, 60^{\circ} \mathrm{C} 30^{\prime \prime}(\mathrm{x} 40)$.

\section{Statistical analysis}

To evaluate the gene expression of mammary epithelial markers we performed the non-parametric Friedman test and Dunn's multiple comparisons tests using GraphPad Prism version 8.2. The differences were considered significant when $\mathrm{p}<0.05$. Unless stated, all values are given as mean \pm standard deviation (SD).

\section{RESULTS}

\section{Evaluation of cells growth in FBS-free medium} grown in FBS-free medium. Cells grew from $\sim 1 \times 10^{5}$ at day 4 to $\sim 1.1 \times 10^{6}$ (day 12) (figure 2a). Coating the plates with collagen I or laminin neither improved cell attachment nor growth during the first days 

did not change compared to FBS containing medium ( $\mathrm{p}>0.05$, table 2$)$.

a)

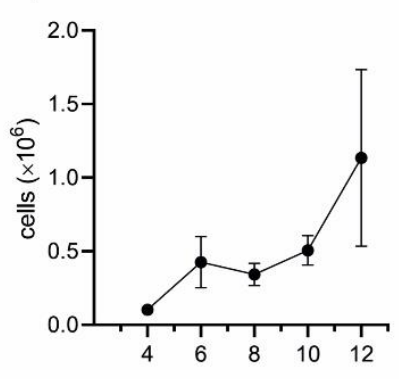

d)

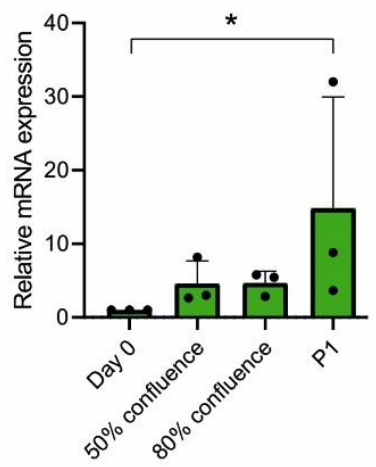

b)

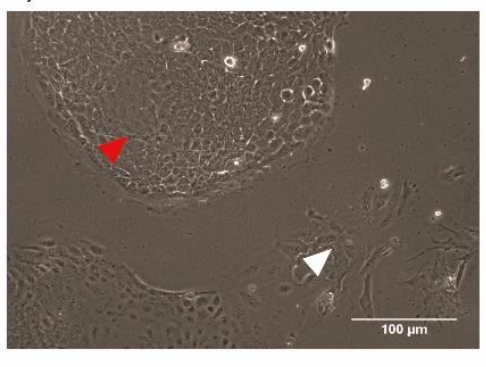

KRT14

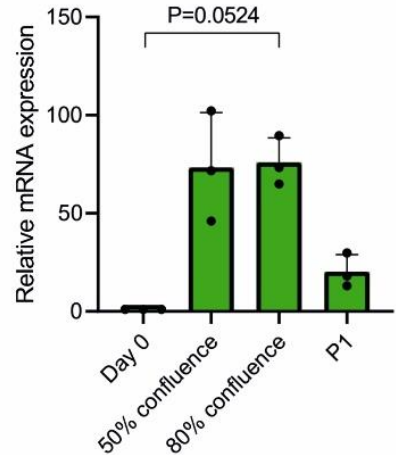

PR c)

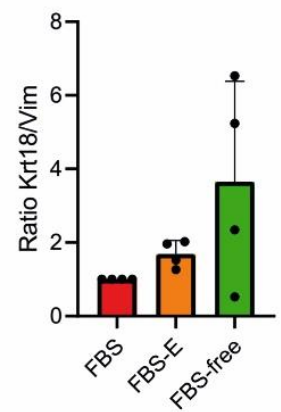

VIM

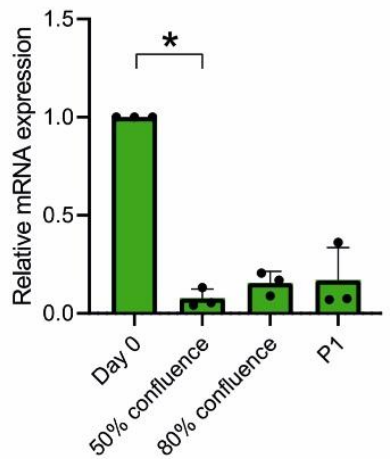

CSN3

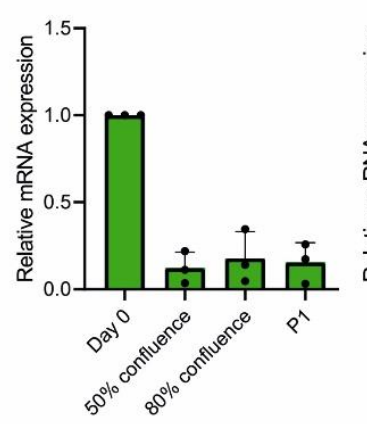

f)

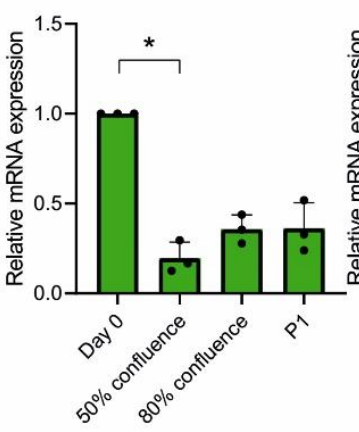

CSN1S1

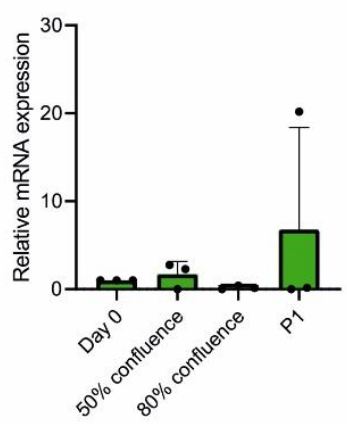

KRT14

VIM
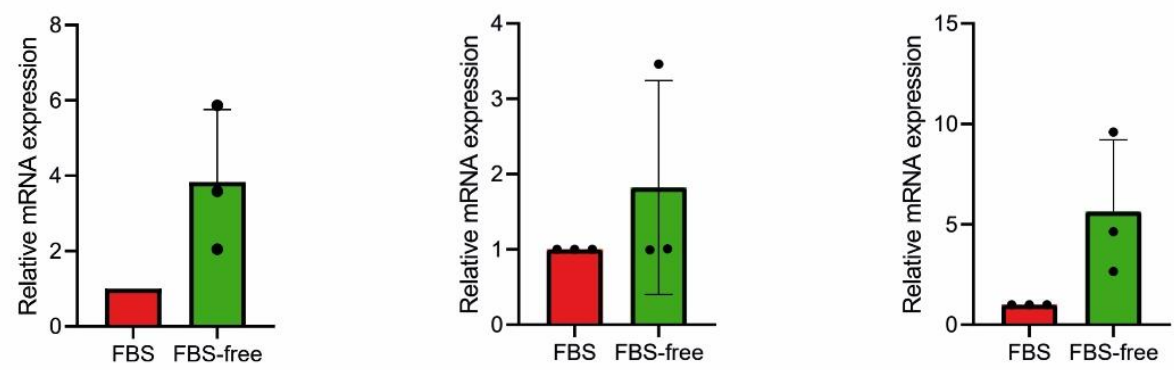
Figure 2. The pbMEC and MAC-T properties in FBS-free medium. a) Cell counts from day 4 to day 12 in 6-wells multiwell culture dishes; b) Epithelial cells population (red arrow), and fibroblast-like cells (white arrow); c,d) Celltype (c) and differentiation (d) markers at the isolation day (day 0), at $50 \%$ and $80 \%$ confluency, and at $80 \%$ confluency at the first sub-passage (P1): keratin 18 (KRT18), keratin 14 (KRT14) for epithelial identity, vimentin (VIM) as fibroblastic marker, prolactin hormone receptor (PRLR), progesterone receptor (PR), casein alpha (CSN1S1) and casein kappa (CSN3) coding genes as differentiation markers; f) Keratin 14 and 18 and vimentin mRNA expression for MAC-T cells cultured in FBS $10 \%$ or FBS-free medium. The mRNA expression data are depicted as $2-\Delta \Delta \mathrm{Ct}$ [42], values are mean $\pm \mathrm{SD}$ of 4 independent replicates, Kruskal-Wallis and Dunn's multiple comparisons tests was performed. The differences were considered significant when $\mathrm{p}<0.05$.

\begin{tabular}{lccc} 
MAC-T cells & Passage $1\left[\mathrm{~h}^{-1}\right]$ & Passage $2\left[\mathrm{~h}^{-1}\right]$ & $\begin{array}{l}212 \\
213\end{array}$ \\
\hline FBS & $0.011 \pm 0.006$ & $0.023 \pm 0.003$ & 214 \\
\hline FBS-free & $0.022 \pm 0.015$ & $0.012 \pm 0.005$ & 215 \\
& & & 216 \\
\hline
\end{tabular}

Table 2. Growth rates of MAC-T grown in FBS containing or FBS-free medium in two consecutive passages. Data are expressed as mean \pm SD of 3 independent experiments. Mann-Whitney test was performed

\section{Evaluation of mammary gland gene markers expression in FBS-free medium}

The primary cultures were initially not pure and were composed of a mixed population of fibroblasts (figure 2b, white arrow) and epithelial cells (figure 2b, red arrow). We evaluated the epithelial cells enrichment as indicated by the gene expression ratio between keratin 18 (an epithelial marker) and vimentin (a fibroblastic marker) in pbMECs cultured in i) FBS 10\% ii) FBS 10\% + pre-plating iii) FBSfree medium. The enrichment of epithelial cells increased 1.7-fold by pre-plating and 3.4-fold in FBS-free medium (figure 2c), compared to pbMECs in FBS without pre-plating. Even if not statistically significant $(p=0.1377$, FBS-free medium vs FBS 10\%), we observed that some cultures reacted to the FBS-free medium by enriching the population in epithelial cells, without the need of pre-plating. Coating the plate with neither collagen I nor laminin significantly affected the enrichment $(p>0.05$, supplementary figure $1 b)$.

Subsequently, we evaluated the gene expression of cell type and differentiation markers at day 0 (isolation day), $50 \%$ and $80 \%$ confluence after day 0 , and at $80 \%$ confluence after the first sub-passage $(\mathrm{P} 1)$. The mRNA abundance of the epithelial markers keratin 14 (KRT14) and 18 (KRT18) increased at 80\% confluency after the isolation day and at $\mathrm{P} 1$, respectively $(\mathrm{p}<0.05$, figure $2 \mathrm{~d})$. On the other hand, the expression of the fibroblastic marker vimentin (VIM) decreased already at $50 \%$ confluence after day 0 $(p<0.05$, figure $2 d)$. The expression of the functional marker progesterone receptor (PR) significantly decreased already at 50\% confluence $(\mathrm{p}<0.05$, figure $2 \mathrm{e})$, while casein alpha (CSN1S1) and prolactin 
238 hormone receptor (PRLR) tended to decrease over time ( $\mathrm{p}>0.05$, figure 2e). Casein kappa (CSN3) did

239 neither significantly change, but the trend was rather to increase in some replicates (figure 2e).

240 MAC-T cells cultured in FBS-free medium presented as a homogeneous population and did not

241 morphologically differ from the FBS-containing environment (supplementary figure 1c). Gene expression

242 of KRT14, KRT18 and VIM neither changed ( $>0.05$, figure $2 \mathrm{f}$ ), indicating that the FBS-free medium did

243 not alter the cell-type composition.

\section{Evaluation of EVs isolation methods from pbMECs and MAC-T conditioning medium}

245 As figure 3a shows, the conditioning FBS-free medium alone did not contain any vesicles, ruling out any

246 contamination of EVs from the medium used. According to the TEM, we obtained particles from all routes

247 except from SEC-UC (figure 3c).

248 Single or aggregates of cup-shaped particles exhibiting the typical EV morphology at TEM [41, 42] were 249 observed following UCx2, UF-SEC-UC, UC-SEC-UC, UC-SEC-UF, UC-SEC-UC, (figure 3b,f,g,h and i;

250 black arrows). The observed structures were heterogeneous in size, ranging from 50 to $500 \mathrm{~nm}$. We also

251 observed light grey aggregates in CP, CP-SEC-UC and UC-SEC-CP (figure 3d, e and g; white asterisk),

252 likely due to lipid aggregates [43, 44], while round non-vesicles [45] were observed from CP, CP-SEC-

253 UC and UF-SEC-UC (figure 3d,_e and f; black asterisk). 
a) FBS-free medium
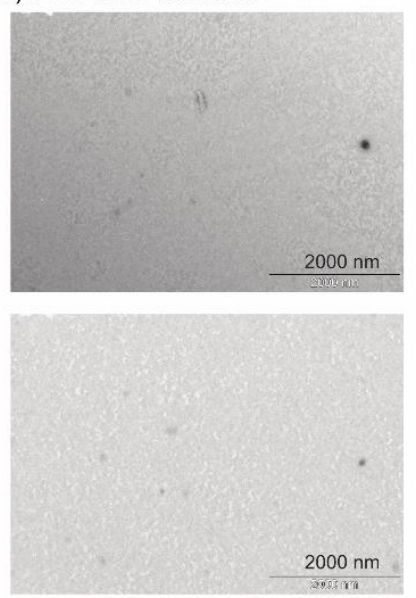

d) $\mathrm{CP}$
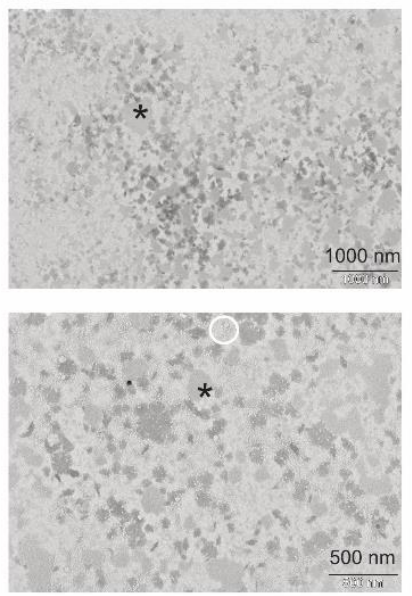

g) UC-SEC-CP
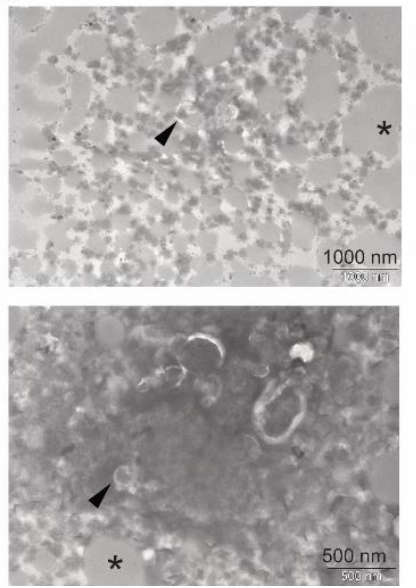

b) UC $\times 2$
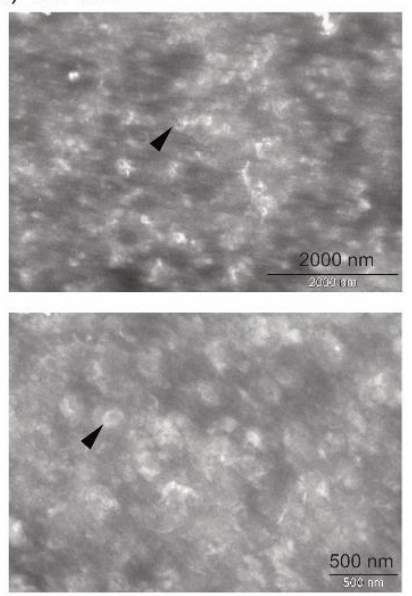

e) CP-SEC-UC
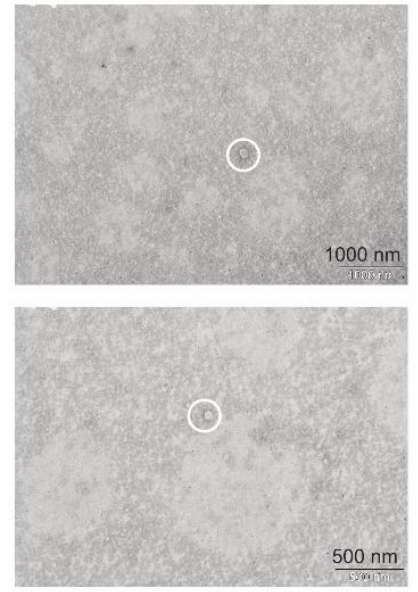

h) UC-SEC-UF
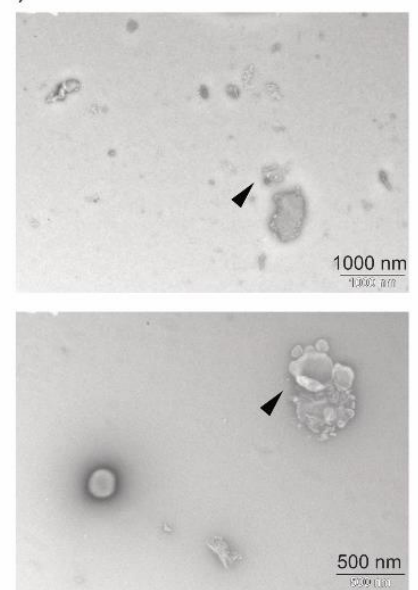

c) SEC-UC
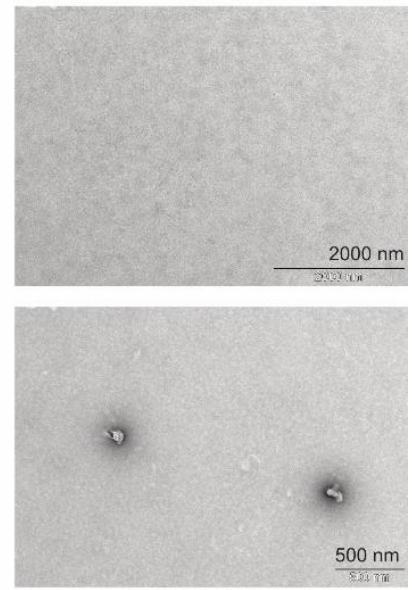

f) UF-SEC-UC
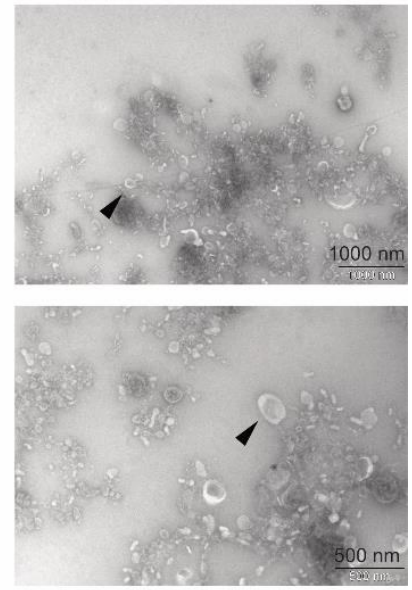

i) UC-SEC-UC
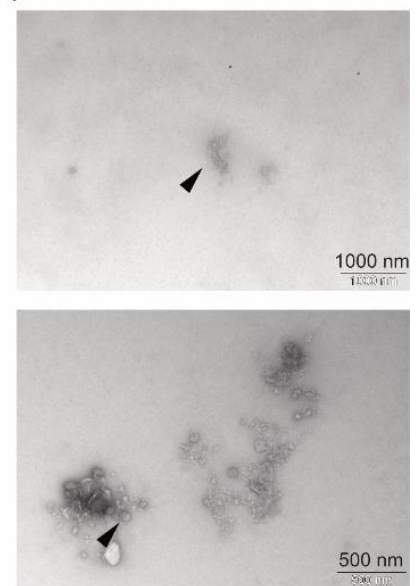

Figure 3. TEM pictures from FBS-free medium (a) and EVs isolated with UC x2 (b), SEC-UC (c), CP (d), CP-SEC-
UC (e), UF-SEC-UC (f), UC-SEC-CP (g), UC-SEC-UF (h), UC-SEC-UC (i). Black arrow: cup-shaped particles 
Table 3 summarizes particle concentration and size measured by tunable resistive pulse sensing (TRPS). nm. We did not detect any particles if following SEC-UC and UC-SEC-UF.

\begin{tabular}{|l|l|l|l|l|l|}
\hline Route & Pellet & Raw & Size range & Mean particle size & Particle rate \\
& resuspension & concentration & & {$[\mathrm{nm}] \pm \mathrm{SD}$} & \\
& volume $[\mu \mathrm{L}]$ & {$[$ particles $/ \mathrm{mL}]$} & & & 192.8 \\
\hline UCx2 & 30 & $4.39 \mathrm{E}+11$ & $67-512$ & $119 \pm 39.7$ & - \\
\hline SEC-UC & 30 & - & - & - & 119.5 \\
\hline CP & 100 & $2.95 \mathrm{E}+09$ & $108-781$ & $195 \pm 70.1$ & 20.02 \\
\hline CP-SEC-UC & 100 & $4.99 \mathrm{E}+08$ & $123-708$ & $210 \pm 89.4$ & 277.4 \\
\hline UF-SEC-UC & 30 & $5.30 \mathrm{E}+10$ & $70-473$ & $139 \pm 36.5$ & 701.2 \\
\hline UC-SEC-CP & 30 & $1.47 \mathrm{E}+10$ & $81-564$ & $151 \pm 70.0$ & - \\
\hline UC-SEC-UF & 150 & - & - & - & 327.0 \\
\hline UC-SEC-UC & 30 & $6.87 \mathrm{E}+09$ & $77-559$ & $158 \pm 60.8$ & \\
\hline
\end{tabular}

Table 3. Summary of TRPS measurements. The data come from one replicate.

The EVs collection routes UCx2, UF-SEC-UC, UC-SEC-CP and UC-SEC-UC yielded a higher particle concentration, in line with the TEM results. The TRPS however did not discriminate between the different kinds of particles [46], such as between vesicles and the undefined aggregates in UC-SEC-CP. Therefore, since UF-SEC-UC and UC-SEC-UC had the cleanest background free of undefined aggregates (figure $3 \mathrm{f}$, i), we selected these two routes to continue with. We obtained similar size distributions (figure 4a) across replicates as well as concentration ( $\mathrm{p}>0.05$, figure $4 \mathrm{~b})$. The last SEC fractions after UF had lower protein concentration than after UC, showing that UF as a first step cleaned better the samples from contaminating proteins (figure 4c).

When isolating EVs from MAC-T with UF-SEC-UC and UC-SEC-UC, we obtained in both routes cup shaped particles as shown with TEM (Figure 4d). The TRPS size and concentration of the isolated particles were 1.63E+E09 and 2.21E+10 particles/mL from UF-SEC-UC and UC-SEC-UC respectively, and similar size ranges (table 4). 

ruling out any intracellular contamination [47] (figure 4e).

a)

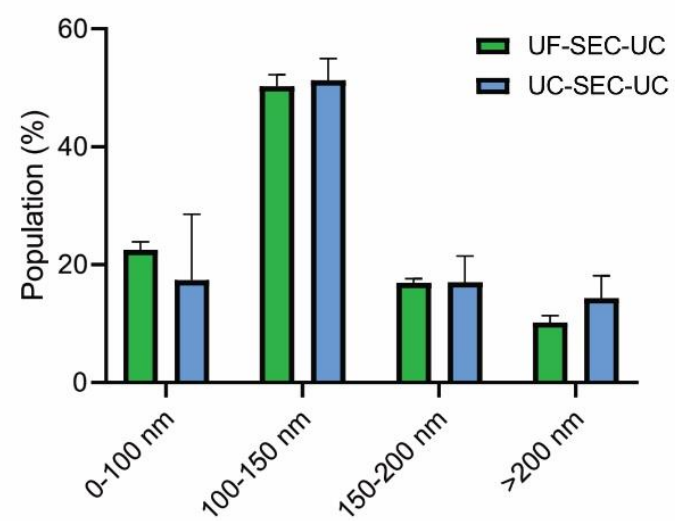

c)

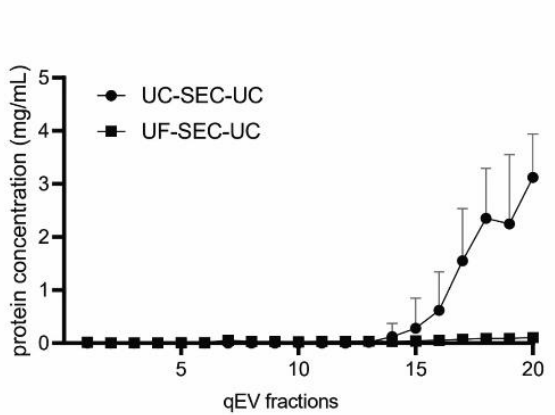

d) UF-SEC-UC (MAC-T)

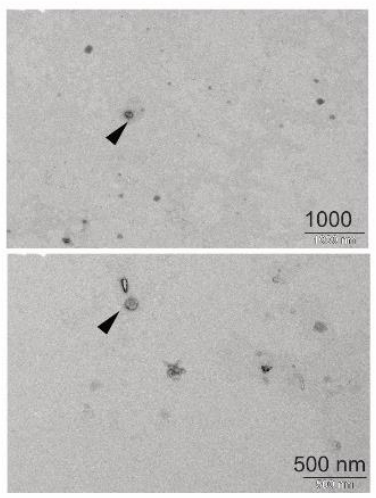

b)

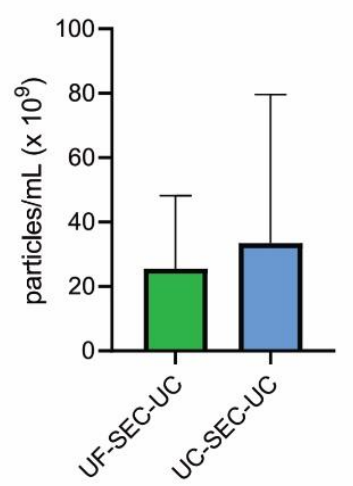

e) UC-SEC-UC (MAC-T)

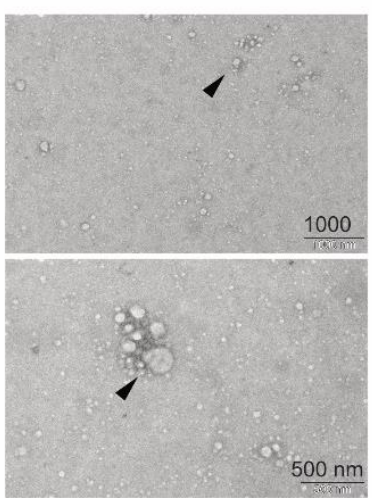

f)

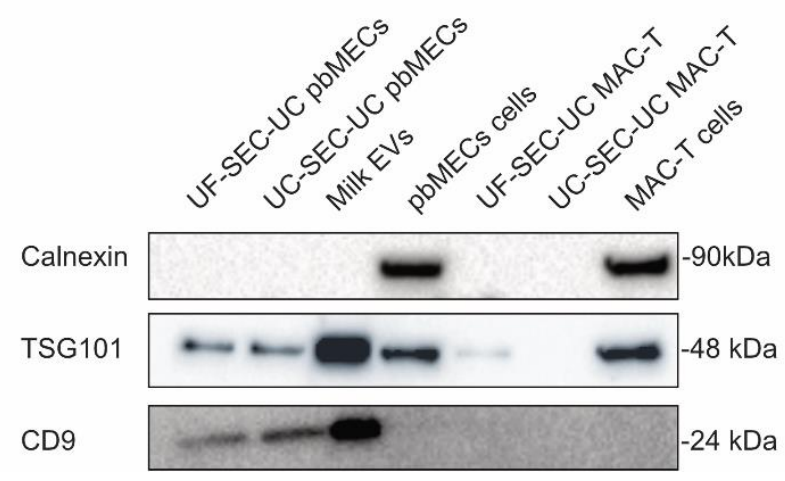

Figure 4. a) Size ranges and b) particle concentration of EVs from UF-SEC-UC and UC-SEC-UC in pbMEC conditioning medium; c) protein concentration of the single fractions from SEC from pbMECs conditioning medium; d-e) TEM pictures after UF-SEC-UC and UC-SEC-UC from MAC-T conditioning medium c) Western blot of pbMECs and MAC-T EVs pellet, whole cells lysates were used as a positive control for calnexin, EVs from milk as a positive control for EV markers. Full-length blots are presented in Supplementary figure 2. In a-c) values are mean \pm SD of three replicates. 


\begin{tabular}{llll} 
Route & Concentration & Diameter range $[\mathrm{nm}]$ & Average particle size [nm] \\
& {$[$ particles $/ \mathrm{mL}]$} & & $\pm \mathrm{SD}$ \\
& & & $135 \pm 60.4$ \\
\hline UF-SEC-UC & $2.24 \mathrm{E}+10$ & $66-593$ & $134 \pm 54.3$
\end{tabular}

c)
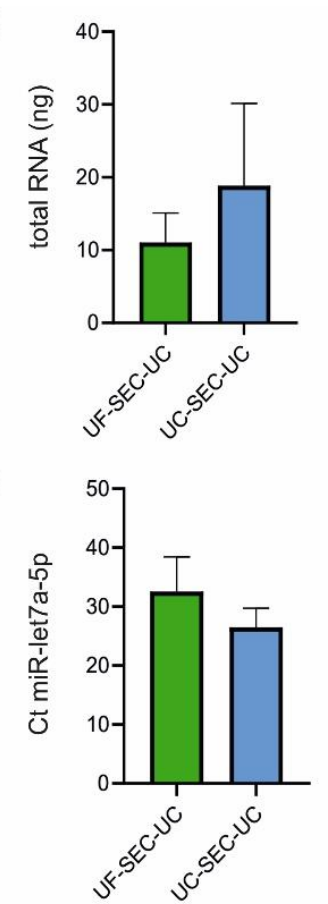

b)

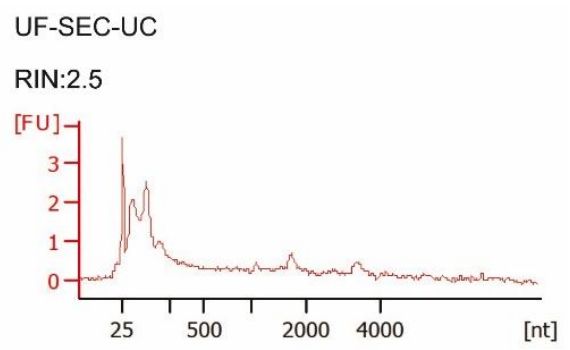

UC-SEC-UC

RIN:3.3

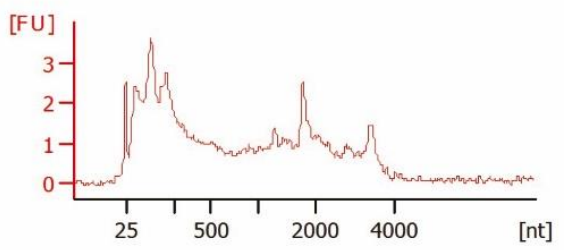

Figure 5. Isolation and characterisation of RNA from pbMEC EV. a) Total amount of RNA from pbMECs EVs isolated with UFSEC-UC or UC-SEC-UC; b) Representative electropherograms from UF-SEC-UC (top), and UC-SEC-UC (bottom); c) Ct of miR- 
304

In the current study, we established for the first time the culture of primary bovine mammary epithelial cells (pbMECs) and MAC-T cells in FBS-free medium to study EVs from the bovine mammary gland in vitro. An important point is that the culture medium is chemically defined, therefore it does not change during the culture and always keeps the same formula, avoiding any possible source of variability given by FBS removal [28, 29] or even different batches of FBS.

Our customized FBS-free medium sustained pbMECs cell growth until confluency and beyond passage 3, as well as the growth of MAC-T cell line. The growth rate of MAC-T was lower than in FBS containing medium. This is in line with previous results, where, however, the culture medium had a different formula [43]. In the primary culture, FBS-free medium promoted the expression of epithelial markers keratin 14 and 18 and the downregulation of the fibroblastic marker vimentin, thereby enriching the population in epithelial cells. In addition, it did not affect the epithelial identity of MAC-T, as keratin 14 and keratin 18 expression levels did not change. Thus, pbMECs and MAC-T cells cultured on FBS free medium were still relatively close to the primary isolated cells at day 0 and FBS-containing medium, respectively.

We also observed a slight downregulation of the differentiation markers PR, PRLR, CSN1S1 and CSN3 already starting at $50 \%$ confluency. This trend to de-differentiate might be owed to the culture on two dimensions (2D) on plastic dishes, which by itself promotes de-differentiation [44, 45]. It has been shown that the growth of pbMECs on tri-dimensional (3D) systems supports differentiation $[37,45,46]$. Thus, further studies should focus on 3D FBS-free culture systems.

The most commonly used techniques to isolate EVs from primary MECs cell cultures or breast cancer lines are dual UC [6, 18, 23, 47, 48] and UF with a cut-off of $100 \mathrm{kDa}$ [17], respectively. By combining these methods, size exclusion chromatography and chemical precipitation, we managed to isolate EVs from relatively low amounts $(10 \mathrm{~mL})$ of cellular conditioning medium, while in the literature, when stated, the reported starting material is often between 20 and $250 \mathrm{~mL}$ [30-32]. The TEM and TRPS measurements excluded any particle contamination in the FBS-free medium, thereby the EVs we observed and analyzed were secreted by the cultured cells. From the eight routes that we tested, only in one route (SEC-UC) we did not isolate any particle after TEM analysis, likely due to the initial low amount of starting material $(500 \mu \mathrm{L})$. Dual UC gave a high amount of particles but the TEM imaging showed that the matrix was not clean, possible due to the low cleaning steps before the UC. The introduction of more differential 
centrifugation steps and a SEC step helped to clean the sample likely from proteins in solution and any membranes or content deriving from cell debris and apoptotic bodies. The miRCURY kit used for CP and CP-SEC-UC instead helped to precipitate vesicles but concomitantly generated many undefined aggregates, as the precipitation itself does not distinguish between the types of macromolecules in solution $[33,49]$. We obtained a clean sample from UC-SEC-UF, but the particles observed after TEM were few in the whole grid, likely due to the high final volume of the sample $(150 \mu \mathrm{L})$, collected from the ultrafiltration tube. Both UF-SEC-UC and UC-SEC-UC revealed a better compromise regarding the yield and purity of vesicles. The TRPS measurements confirmed the size ranges observed at TEM and were similar between routes, and both gave a consistent amount of EVs, on the order of 109 -1010 particles/mL. Western blot analysis confirmed that the isolated vesicles were actual EV, bearing both CD9 and TSG101, in line with the results obtained by Zang et al. [17], and from studies on milk EVs [19]. Importantly, the use of a SEC step to separate secreted proteins from EVs would allow analysing the cell response distinguishing the contribution of EVs and secreted proteins. For such purpose, UC-SEC-UC would be more suitable, as the protein-rich fractions are more concentrated, without the initial UF step. On the other hand, if the experimental setup requires the study of EVs only, UF-SEC-UC would be more recommended, as the sample is initially depleted from proteins smaller than $100 \mathrm{k} \mathrm{kDa}$.

We were able to extract enough RNA and detect miR-let-7a-5p after using both protocols. MiR-let-7a-5p is a miRNA expressed in the bovine mammary gland tissue [50] and is one of the most abundant exosomal miRNAs found in both human and bovine milk [51]. We observed a higher amount of RNA and miR-let7a-5p with UC-SEC-UC, however that protocol gave higher contamination from rRNA 18S and 28S, likely pulled down with the first ultracentrifugation step [52].

In conclusion, we demonstrated that the FBS-free medium culture system is a valid tool to study MECs EVs from both primary cells and the MAC-T cell line. We evaluated and compared different EVs isolation protocols from a relatively low amount of starting cell culture medium $(10 \mathrm{~mL})$ and we proposed both UCSEC-UF and UC-SEC-UC are the preferred methods to isolate the highest amount of pure EVs for further downstream analysis. Our results provide an important reference for further studies that aim at analysing MECs EVs in many contexts such as lactation, infection, response to stressors and metabolic challenges. 


\section{DECLARATIONS}

\section{Acknowledgements}

362 We thank S. Handschin from the Scientific Center for Optical and Electron Microscopy (ScopeM) of ETH

363 Zurich for his support with TEM. The authors are active participants of the COST Action CA16119 (In

364 vitro 3D total cell guidance and fitness).

\section{Authors' contributions}

366 GS conceptualized the experiments, performed the in vitro cultures, EVs isolation, analysed the data and wrote the manuscript. SEU supervised and financed the project and revised the manuscript. MDS the final manuscript.

\section{Availability of data and materials}

371 Not applicable.

$372 \quad$ Financial support and sponsorship

373 This work was supported by the ETH Research Grant (ETH-53 16-1).

\section{Conflicts of interest}

375 All authors declared that there are no conflicts of interest.

\section{Ethical approval and consent to participate}

377 Not applicable.

\section{Consent for publication}

379 Not applicable.

\section{Copyright}

381 (c) The Author(s) 2020. 


\section{REFERENCES}

1. Lin, M.C., et al., Transfer of mammary Gland-Forming ability between mammary basal epithelial cells and mammary luminal cells via extracellular vesicles/exosomes. Journal of Visualized Experiments, 2017.

2. Nakatani, H., et al., Weaning-induced expression of a milk-fat globule protein, MFG-E8, in mouse mammary glands, as demonstrated by the analyses of its $m R N A$, protein and phosphatidylserinebinding activity. Biochemical Journal, 2006.

3. Chin, A.R., et al., Polarized Secretion of Extracellular Vesicles by Mammary Epithelia. Journal of Mammary Gland Biology and Neoplasia, 2018.

4. Sun, J., et al., MicroRNA expression profiles of bovine milk exosomes in response to Staphylococcus aureus infection. BMC Genomics, 2015.

5. Cai, M., et al., Genome-wide microRNA profiling of bovine milk-derived exosomes infected with Staphylococcus aureus. 2018: p. 663-672.

6. Dutta, S., et al., Interactions between exosomes from breast cancer cells and primary mammary epithelial cells leads to generation of reactive oxygen species which induce DNA damage response, stabilization of 53 and autophagy in epithelial cells. PloS one, 2014. 9(5).

7. Steinbichler, T.B., et al. The role of exosomes in cancer metastasis. in Seminars in cancer biology. 2017. Elsevier.

8. Chen, W.x., et al., Exosomes from drug-resistant breast cancer cells transmit chemoresistance by a horizontal transfer of microRNAs. PloS one, 2014.

9. $\quad$ Dong, X., et al., Exosomes and breast cancer drug resistance. Cell death \& disease, 2020. 11(11): p. 1-14.

10. Admyre, C., et al., Exosomes with immune modulatory features are present in human breast milk. The Journal of immunology, 2007. 179(3): p. 1969-1978.

11. E. Sedykh, S., et al., Milk Exosomes: Isolation, Biochemistry, Morphology, and Perspectives of Use, in Extracellular Vesicles [Working Title]. 2019.

12. Benmoussa, A., et al., Commercial dairy cow milk microRNAs resist digestion under simulated gastrointestinal tract conditions. The Journal of nutrition, 2016. 146(11): p. 2206-2215.

13. Chen, Z., et al., Milk exosome-derived miRNAs from water buffalo are implicated in immune response and metabolism process. BMC Veterinary Research, 2020. 16: p. 1-5.

14. Zhu, Z., et al., Goat milk-derived exosomes Endowed with Radioactive and Targeting Properties: potential to provide PET/CT monitoring for exosomes-based drug delivery system in gliomas therapy. Journal of Nuclear Medicine, 2020. 61(supplement 1): p. 1064-1064.

15. Hendrix, A.N. and A.N. Hume, Exosome signaling in mammary gland development and cancer. 2011: p. 879-887.

16. Lowry, M.C., W.M. Gallagher, and L. O'Driscoll, The role of exosomes in breast cancer. Clinical chemistry, 2015. 61(12): p. 1457-1465.

17. Zhang, M., et al., Proteomic analysis reveals proteins and pathways associated with lactation in bovine mammary epithelial cell-derived exosomes. Journal of Proteome Research, 2020.

18. Huang, T., et al., Exosomes Derived from Bovine Mammary Epithelial Cells Treated with Transforming Growth Factor- $\beta 1$ Inhibit the Proliferation of Bovine Macrophages. Journal of Interferon \& Cytokine Research, 2019. 39(12): p. 752-759.

19. Benmoussa, A., et al., Identification of protein markers for extracellular vesicle (EV) subsets in 
cow's milk. Journal of Proteomics, 2019.

20. Benmoussa, A., et al., A subset of extracellular vesicles carries the bulk of microRNAs in commercial dairy cow's milk. Journal of Extracellular Vesicles, 2017. 6: p. 1-22.

21. Weaver, S.R. and L.L. Hernandez, Autocrine-paracrine regulation of the mammary gland. Journal of Dairy Science, 2016.

22. Lehrich, B.M., et al., Fetal Bovine Serum-Derived Extracellular Vesicles Persist within VesicleDepleted Culture Media. International journal of molecular sciences, 2018. 19.

23. Chen, Y., et al., Transcriptional Profiling of Exosomes Derived from Staphylococcus aureusInfected Bovine Mammary Epithelial Cell Line MAC-T by RNA-Seq Analysis. Oxidative Medicine and Cellular Longevity, 2021. 2021.

24. Eitan, E., et al., Extracellular vesicle-depleted fetal bovine and human sera have reduced capacity to support cell growth. Journal of extracellular vesicles, 2015. 4(1): p. 26373.

25. Angelini, F., et al., Foetal bovine serum-derived exosomes affect yield and phenotype of human cardiac progenitor cell culture. BioImpacts: BI, 2016. 6(1): p. 15.

26. Beninson, L.A. and M. Fleshner, Exosomes in fetal bovine serum dampen primary macrophage $I L-1 \beta$ response to lipopolysaccharide (LPS) challenge. Immunology letters, 2015. 163(2): p. 187192.

27. Liao, Z., et al., Serum extracellular vesicle depletion processes affect release and infectivity of HIV-1 in culture. Scientific Reports, 2017.

28. Shin, J.-S., et al., Serum starvation induces G1 arrest through suppression of Skp2-CDK2 and CDK4 in SK-OV-3 cells. International journal of oncology, 2008. 32(2): p. 435-439.

29. Chowdhury, P., S. Sacks, and N. Sheerin, Toll-like receptors TLR2 and TLR4 initiate the innate immune response of the renal tubular epithelium to bacterial products. Clinical \& Experimental Immunology, 2006. 145(2): p. 346-356.

30. Arellano-Anaya, Z.E., et al., Prion strains are differentially released through the exosomal pathway. Cellular and molecular life sciences, 2015. 72(6): p. 1185-1196.

31. Mitchell, J.P., et al., Increased exosome production from tumour cell cultures using the Integra CELLine Culture System. Journal of immunological methods, 2008. 335(1-2): p. 98-105.

32. Lin, R., S. Wang, and R.C. Zhao, Exosomes from human adipose-derived mesenchymal stem cells promote migration through Wnt signaling pathway in a breast cancer cell model. Molecular and cellular biochemistry, 2013. 383(1-2): p. 13-20.

33. Patel, G.K., et al., Comparative analysis of exosome isolation methods using culture supernatant for optimum yield, purity and downstream applications. Scientific reports, 2019. 9(1): p. 1-10.

34. Zavizion, B., et al., Establishment and characterization of a bovine mammary epithelial cell line with unique properties. In Vitro Cellular \& Developmental Biology-Animal, 1996. 32(3): p. 138148.

35. Huynh, H.T., G. Robitaille, and J.D. Turner, Establishment of bovine mammary epithelial cells (MAC-T): an in vitro model for bovine lactation. Experimental cell research, 1991. 197(2): p. 191-199.

36. Wellnitz, O. and D.E. Kerr, Cryopreserved bovine mammary cells to model epithelial response to infection. Veterinary immunology and immunopathology, 2004. 101(3-4): p. 191-202.

37. Hillreiner, M., et al., Establishment of a $3 D$ cell culture model of primary bovine mammary epithelial cells extracted from fresh milk. 2017: p. 706-720.

38. Heo, Y.T., et al., Mammary alveolar cell as in vitro evaluation system for casein gene expression 
involved in glucose level. Asian-Australasian journal of animal sciences, 2017. 30(6): p. 878.

39. German, T. and I. Barash, Characterization of an epithelial cell line from bovine mammary gland. In Vitro Cellular \& Developmental Biology-Animal, 2002. 38(5): p. 282-292.

40. Pan, C., et al., Comparative proteomic phenotyping of cell lines and primary cells to assess preservation of cell type-specific functions. Molecular \& Cellular Proteomics, 2009. 8(3): p. 443450.

41. Finot, L., E. Chanat, and F. Dessauge, Molecular signature of the putative stem / progenitor cells committed to the development of the bovine mammary gland at puberty. Scientific Reports, 2018: p. 1-11.

42. Livak, K.J. and T.D. Schmittgen, Analysis of relative gene expression data using real-time quantitative PCR and the 2- $\triangle \triangle C T$ method. methods, 2001. 25(4): p. 402-408.

43. Zavizion, B., R. Gorewit, and I. Politis, Subcloning the MAC-T bovine mammary epithelial cell line: morphology, growth properties, and cytogenetic analysis of clonal cells. Journal of dairy science, 1995. 78(3): p. 515-527.

44. Pampaloni, F., E.G. Reynaud, and E.H.K. Stelzer, The third dimension bridges the gap between cell culture and live tissue. 2007. 8: p. 839-845.

45. Turrubiarte, M.A., et al., Phenotypic and functional characterization of two bovine mammary epithelial cell lines in 2D and 3D models. 2018: p. 348-356.

46. Walter, L., et al., Investigation on the suitability of milk-derived primary bovine mammary epithelial cells grown on permeable membrane supports as an in vitro model for lactation. In Vitro Cellular \& Developmental biology. Animal, 2020.

47. Jenjaroenpun, P., et al., Characterization of RNA in exosomes secreted by human breast cancer cell lines using next-generation sequencing. PeerJ, 2013. 1: p. e201.

48. O'Brien, K., et al., Exosomes from triple-negative breast cancer cells can transfer phenotypic traits representing their cells of origin to secondary cells. European journal of cancer, 2013. 49(8): p. 1845-1859.

49. Karttunen, J., et al., Precipitation-based extracellular vesicle isolation from rat plasma coprecipitate vesicle-free microRNAs. Journal of extracellular vesicles, 2019. 8(1): p. 1555410.

50. Wicik, Z., et al., Characterization of micro RNA profile in mammary tissue of dairy and beef breed heifers. Journal of Animal Breeding and Genetics, 2016. 133(1): p. 31-42.

51. Özdemir, S., Identification and comparison of exosomal microRNAs in the milk and colostrum of two different cow breeds. Gene, 2020. 743: p. 144609.

52. Tang, Y.-T., et al., Comparison of isolation methods of exosomes and exosomal RNA from cell culture medium and serum. International journal of molecular medicine, 2017. 40(3): p. 834-844. 